\title{
La especialización productiva y agrícola desde su análisis bibliométrico (1915-2019)
}

Victoria Pacheco-Almaraz*, María Isabel Palacios-Rangel*, Enrique Genaro Martínez-González*, Juan Manuel Vargas-Canales**, Jorge G. Ocampo-Ledesma*

*Centro de Investigaciones Económicas, Sociales y Tecnológicas de la Agroindustria y la Agricultura Mundial (CIESTAAM), Universidad Autónoma Chapingo. (México)

Correo-e: vpacheco@ciestaam.edu.mx | ORCID iD: https://orcid.org/0000-0002-9825-1566

Correo-e: marisapalacios@gmail.com | ORCID iD: https://orcid.org/0000-0001-9382-863X

Correo-e: enriquemartinez@ciestaam.edu.mx | ORCID iD: https://orcid.org/0000-0001-9312-5002

Correo-e: jocampo@ciestaam.edu.mx | ORCID iD: https://orcid.org/0000-0001-5272-720X

**Departamento de Estudios Sociales. División de Ciencias Sociales y Administrativas Campus Celaya-Salvatierra.

Universidad de Guanajuato. (México)

Correo-e: jm.vargas@ugto.mx | ORCID iD: https://orcid.org/ORCID: 0000-0003-1918-9395

Recibido: 23-04-20; 2a versión: 06-08-20; Aceptado: 26-09-20; Publicado: 16/07/2021

Cómo citar este artículo/Citation: Pacheco-Almaraz, V.; Palacios-Rangel, M. I., Martínez-González, E., Vargas-Canales, J. M., Ocampo-Ledesma, J. G. (2021) La especialización productiva y agrícola desde su análisis bibliométrico (1915-2019). Revista Española de Documentación Científica, 44 (3), e304. https://doi.org/10.3989/redc.2021.3.1764

Resumen: La creciente importancia de la planificación regional genera que la geografía económica y el análisis espacial, así como la producción científica en torno al tema, tomen relevancia. En ese sentido, se realizó un análisis bibliométrico basado en veintiséis conceptos relacionados con "productive specialization" identificando relaciones entre términos y áreas temáticas en dicha categoría de análisis, para el periodo 1915-2019. Se analizaron 1764 documentos obtenidos de Scopus, mediante estadística descriptiva y el software VOSviewer, con técnicas bibliométricas. Se encontró que las líneas que más estudian estos conceptos son especialización regional y crecimiento económico, neurociencia en humanos y animales y producción y prácticas agrícolas. Además, existe un aumento progresivo en la proporción de artículos con mayor colaboración entre autores y países que abordan el tema. Se concluye que son múltiples los sectores estudiados a través de los conceptos planteados, pero destaca el industrial, generando vacíos de conocimiento en el agropecuario.

Palabras clave: Localización económica; economías de aglomeración; técnicas de análisis regional; técnicas bibliométricas; análisis clúster.

\section{Productive and Agricultural Specialization from its bibliometric analysis (1915-2019)}

Abstract: The growing importance of regional planning makes economic geography and spatial analysis, as well as scientific production on the subject, become relevant. In this sense, a bibliometric analysis based on twenty-six concepts related to "productive specialization" was carried out, identifying relationships between terms and thematic areas in said category of analysis, for the period 1915-2019. All 1764 documents obtained from Scopus were analyzed using descriptive statistics and VOSviewer software, with bibliometric techniques. It was found that the most frequent lines that study these concepts are regional specialization and economic growth, neuroscience in humans and animals, and agricultural production and practices. Furthermore, there is a progressive increase in the proportion of articles with greater collaboration between authors and countries that address the subject. It is concluded that there are multiple sectors studied through the concepts raised, but the industrial sector stands out, generating knowledge gaps in agriculture.

Key words: Economic location; economies of agglomeration; regional analysis techniques; bibliometric techniques; cluster analysis.

Copyright: (c) 2021 CSIC. Este es un artículo de acceso abierto distribuido bajo los términos de la licencia de uso y distribución Creative Commons Reconocimiento 4.0 Internacional (CC BY 4.0). 


\section{INTRODUCCIÓN}

La creciente importancia de la planificación regional ha generado que la geografía económica y el análisis espacial tomen relevancia como métodos de análisis de las dinámicas productivas de los países, particularmente porque la comprensión de asimetrías regionales puede mejorar la toma de decisiones relacionadas con inversión, procesos productivos e inequidad y canalizar a un estado superior de desarrollo, con políticas públicas que promuevan éste y el bienestar.

Esta idea se remonta al contexto del Distrito Industrial en el que de acuerdo con Marshall (1920), el desarrollo y configuración de la economía depende de una correlación entre factores internos, (expresados a partir de las condiciones de complementariedad territorial), que inciden en su capacidad operativa y eficiencia productiva; y externos, que le posibilitan el crecimiento de su capacidad de expansión mercantil, factores que en otro escenario delimitan a la empresa (Garofoli, 1986). En relación con la importancia que tienen los atributos contenidos en el espacio geográfico, Becattini (2006) destaca que éstos se vinculan con el desarrollo de modelos de eficiencia industrial y crecimiento empresarial. También demuestra que la localización de las actividades productivas depende del conjunto de externalidades o economías externas complementarias que cada territorio posee, y se convierten en factor determinante en la capacidad de aglomeración de las actividades productivas.

Los estudios realizados en torno a esta temática forman parte del conjunto de productos generados a través de actividades vinculadas a la investigación, desarrolladas por científicos e investigadores; que, de acuerdo con Jiménez (1993), se denominan producción científica; y dentro del mismo se encuentran artículos, libros y capítulos de libros. Respecto a la literatura científica, a partir del siglo $X X$, se consolida la aplicación de métodos matemáticos y estadísticos para su estudio, formando parte central de la bibliometría, que revela información sobresaliente de las publicaciones científicas a través de diferentes indicadores. En ese sentido, los indicadores bibliométricos conforman datos estadísticos deducidos de las publicaciones científicas (Gómez y Bordons, 1996) y pueden clasificarse de acuerdo con términos estadísticos como indicadores unidimensionales y multidimensionales o relacionales (Sanz Casado, 2000). De acuerdo con este autor, estos últimos estudian simultáneamente varias características, estableciendo múltiples interrelaciones en las publicaciones o en los hábitos de investigación de los científicos. Se destaca que los resultados de tales análisis pueden representarse gráficamente a través de mapas bibliométricos, que ofrecen datos sobre relaciones cognitivas y sociales o a través del análisis de redes (Abbasi y Altmann, 2010).

Relacionando el tema de interés con la literatura científica existente en torno a éste, los estudios para determinar la dinámica originada por la distribución espacial de la producción, se han enfocado en el análisis de economías concentradas, específicamente en la especialización y localización de la actividad, mediante el uso de indicadores que contrastan diferentes tipologías de producción de un territorio asociado, así como del total del país. En ese sentido, se han construido índices sintéticos con base en planteamientos pioneros de Boisier (1980), destacando trabajos de Arias y Fortich (2010), Qin y Zhang (2016) y Rebollar y otros (2016), entre varios autores, cuyos indicadores ilustran de manera efectiva la ruta de orientación de las unidades económicas. Para el caso de México, donde el gobierno tiene como una de sus estrategias de política agropecuaria, la promoción del impulso de cadenas agroalimentarias que favorecen el desarrollo de regiones con Especialización Productiva, es necesario analizar si existen publicaciones que reflejan estas experiencias, así como tendencias en las mismas.

Por ello, el objetivo de la presente contribución fue identificar las líneas de investigación actuales sobre Especialización Productiva ubicadas en la frontera del conocimiento, mediante un análisis bibliométrico, para comprender áreas de estudio, autores y fuentes influyentes con publicaciones sobre el tema durante el periodo 1915-2019. Se parte de la hipótesis que los estudios relacionados con Especialización Productiva se enfocan mayormente en el sector industrial y pecuario, generando vacíos de conocimiento en cuanto al análisis de la dinámica productiva agrícola a nivel producto y región, esto último, particularmente en el caso de México.

Para tal fin, esta contribución se compone de una introducción, seguida de materiales y métodos, donde se aclara la cantidad de documentos analizados y fuentes de información. Posteriormente, se presenta el análisis descriptivo de los resultados, así como información generada de la revisión sistemática, utilizando técnicas bibliométricas como co-ocurrencias, co-citación y emparejamiento bibliográfico. Por último, se señalan las conclusiones y referencias.

\section{MATERIALES Y MÉTODOS}

El procedimiento seguido consistió en tres etapas: 1) selección de fuentes de información y reco- 
pilación de datos; 2) determinación de indicadores y procesamiento de las unidades de análisis; y 3) visualización de resultados. Se utilizó una base de datos obtenida de Scopus y para generar, agrupar y visualizar redes se empleó el software VOSviewer (Van Eck y Waltman, 2010).

\section{Selección de fuentes de información y recopi- lación de datos}

La identificación de los documentos se realizó durante el segundo semestre de 2019, a través de la base de datos Scopus, planteando una estrategia de búsqueda limitada a "tema" (título, abstract, palabras clave), con la combinación de palabras clave para precisar de mejor manera la búsqueda. Dichas palabras clave estaban asociadas al término Especialización Productiva ("Productive specialization", "specialization of production", "specialization of the production", "specialization in production", "specialization in the production", "agricultural specialization", "specialization in agriculture", "specialization in the agriculture", "specialization of agriculture", "specialization of the agriculture", "specialization" AND "agricultural production", "specialization" AND "crop production", "sectorial specialization", "regional specialization", "economic specialization", "industrial specialization", "commercial specialization", "job specialization", "specialization" AND "animal production", "specialization" AND "livestock production", "specialization" AND "forest production", "specialization" AND "horticulture production", "specialization" AND "mining production", "specialization" AND "industrial production", "specialization" AND "agricultural cluster" y "specialization of farms") que se conectaron mediante el operador booleano "or". Así mismo, se colocó un filtro por tipo de documentos, eligiendo únicamente los presentados en forma de artículos, con un rango temporal de 1915 hasta diciembre 2019, obteniéndose un total de 1776 documentos.

Cabe señalar que se eliminaron aquellos documentos repetidos (8) y sin datos de autor (4), resultando un total de 1764 contribuciones como base para el análisis bibliométrico; de los cuales se analizaron los resúmenes, títulos y palabras clave. Es importante señalar que el método es de carácter descriptivo y se realiza a partir del análisis de los artículos publicados (Montero y León, 2007), con una descripción sistemática de un conjunto de indicadores generales y otros asociados tanto a autores como a revistas. Se empleó la base de Scopus por ser la más utilizada en revisiones con análisis bibliométrico y la base de datos de citas y resúmenes más grande de literatura revisada por pares (Md Khudzari y otros, 2018).

\section{Determinación de indicadores y procesamien- to de las unidades de análisis}

Para el procesamiento y análisis descriptivo de los documentos, basados en Sanz (2000), se utilizaron una serie de indicadores unidimensionales que describen las principales características de éstos, mismos que se resumen en la Tabla I.

Tabla I. Indicadores analizados

\begin{tabular}{|c|c|}
\hline Indicadores & Descripción \\
\hline \multicolumn{2}{|l|}{ Indicadores de producción } \\
\hline Número de documentos & $\begin{array}{l}\text { Número de artículos publi- } \\
\text { cados en Scopus }\end{array}$ \\
\hline Idioma & $\begin{array}{l}\text { Idioma de publicación: in- } \\
\text { glés, español, ruso, fran- } \\
\text { cés, alemán, etc. }\end{array}$ \\
\hline Área de estudio & $\begin{array}{l}\text { Área de estudio: Ciencias } \\
\text { sociales, Economía, eco- } \\
\text { nometría y finanzas, etc. }\end{array}$ \\
\hline Género & Femenino o masculino \\
\hline \multicolumn{2}{|l|}{ Indicadores de colaboración } \\
\hline $\begin{array}{l}\text { Número publicaciones por } \\
\text { periodos }\end{array}$ & $\begin{array}{l}\text { Número de publicaciones } \\
\text { por periodos en función } \\
\text { del número de autores por } \\
\text { artículo }\end{array}$ \\
\hline Países que publican & $\begin{array}{l}\text { Países que publican sobre } \\
\text { el tema en cuestión }\end{array}$ \\
\hline \multicolumn{2}{|l|}{ Indicadores de tendencia } \\
\hline Número de publicaciones & Publicaciones por año \\
\hline
\end{tabular}

Después de la caracterización de los artículos, se procedió a realizar el análisis clúster con el Software VOSviewer, con la técnica bibliométrica co-ocurrencias, basada en el análisis de las coincidencias de términos, lo que permite la descripción de la investigación más avanzada, produciendo un mapeo con las relaciones entre diversos términos y su asociación en grupos temáticos. Esta técnica, se realiza a través de minería de texto para identificar palabras en texto o con la herramienta de co-palabras (sus unidades de análisis pueden ser todas las palabras tomadas del título, resumen y palabras clave, palabras clave del autor o palabras clave de índice, respectivamente) y se presenta en dos palabras que aparecen simultáneamente en el mismo documento. La medida del enlace entre dos palabras de una red es proporcional a la co-ocurrencia de esas dos palabras en el conjunto de documentos (Ortega-Priego y Aguillo, 2006).

Cabe señalar que para esta técnica se utilizaron todos los términos contenidos en títulos, resúmenes y palabras clave de los 1764 artículos publi- 
cados hasta la actualidad, para abordar los temas principales de la investigación en Especialización Productiva. En un primer momento se eligió el método de conteo binario, donde sólo se identifica la presencia o ausencia de ese término en el documento, sin considerar su número de apariciones. Con la elección de este método se identificaron 8767 términos. Pero después de definir un número mínimo de ocurrencias para facilitar la visualización de redes (treinta), se obtuvieron 51 que, finalmente con la eliminación de términos irrelevantes, conformaron un listado de 34 (es decir, el 66\% del total), lo que permitió hacer la caracterización de cada grupo al considerar el número de ocurrencias de términos dentro del mismo, su interrelación y localización espacial. De igual forma, se detallan algunos aspectos del material que describe los grupos temáticos.

Respecto a la co-citación, ésta se da en dos unidades de análisis (referencias, fuentes y autores) que son citadas por otros documentos publicados con posterioridad a ellos, denotando de esta manera un enfoque progresivo (Garfield, 1998). Cabe señalar que para esta técnica se emplearon fuentes y autores como unidades de análisis, y para el caso de las revistas, se destaca que de un total de 29.174 para la construcción de la red de co-citación se estableció un punto de corte $\geq 30$; es decir, se seleccionaron exclusivamente las fuentes citadas al menos treinta veces, obteniéndose un total de 177 . Se aclara que para facilitar la visualización de la red, en ésta únicamente se señalan las más representativas del campo, que representan el $50 \%$ del total de fuentes; de manera adicional, para algunas revistas se presentan los indicadores de impacto de acuerdo con el valor del Cite Score de Scopus (ELSEVIER, 2016) y Journal Impact Factor (JIF) recuperado de Clarivate Analytics desde Web of Science (WoS), que es el número promedio de veces que los artículos publicados en una revista específica en los dos años anteriores fueron citados en un año en particular (Thomson, 2019).

Para la construcción de la red de co-citación de autores, de los 67.301 citados se estableció un punto de corte $\geq 30$, seleccionando aquéllos que han sido citados al menos 30 veces, obteniendo un listado de 138 , que responden a los más representativos del campo, y de los cuales para algunos casos se presenta el índice H propuesto por Hirsch (2005), que plantea que cada trabajo elaborado por un autor se ordena de forma descendente en relación con el número de citas recibidas, a lo que se anexa el número de orden que tiene el trabajo en un ranking (o rango) determinado. A partir de esto se construyen dos listas de números: una descendente que contiene el número de citas recibidas por el trabajo, y otra ascendente que presenta el ranking o rango que le corresponde, de esta forma, cuando ambos valores se cruzan se forma dicho índice.

Cabe señalar que, en el análisis del listado de autores, los enlaces mostraron las relaciones de co-citación entre cada dos autores, y el color de los nodos distinguió el grupo al que cada uno estuvo asociado por su similitud temática. Los nodos de mayor tamaño se vincularon con los que recibieron un mayor número citas.

Por último, el emparejamiento bibliográfico se aplicó a países cuyas producciones tienen referencias bibliográficas comunes, con la finalidad de mostrar la participación de México en estudios con el tema de interés. En este caso, de un total de 130 países, para la construcción de la red se estableció un punto de corte de cinco documentos por país, pero con veinte citaciones por país, es decir, se seleccionaron exclusivamente los países citados al menos veinte veces, obteniendo de esta forma un listado de 45 naciones, que representan el 34\% de países y aproximadamente el $13 \%$ de los documentos. La principal diferencia con la co-citación, es que en este caso existe una relación estable y permanente y depende de las referencias contenidas en estos documentos, mientras que en la co-citación la relación entre dos documentos varía, dependiendo del número de veces que son citados por documentos posteriores a su publicación (Garfield, 1998). Un resumen de las técnicas bibliométricas empleadas se presenta en Tabla II.

Tabla II. Tipos de análisis seleccionados

\begin{tabular}{|l|l|l|}
\hline \multicolumn{1}{|c|}{$\begin{array}{c}\text { Tipo de } \\
\text { análisis }\end{array}$} & \multicolumn{1}{|c|}{ Objetivo } & \multicolumn{1}{|c|}{$\begin{array}{c}\text { Unidad de } \\
\text { análisis }\end{array}$} \\
\hline Co-ocurrencia & $\begin{array}{l}\text { Identificar líneas } \\
\text { temáticas de } \\
\text { investigación }\end{array}$ & $\begin{array}{l}\text { Todos los } \\
\text { términos } \\
\text { de títulos, } \\
\text { resúmenes y } \\
\text { palabras clave }\end{array}$ \\
\hline Co-citación & $\begin{array}{l}\text { Aproximar base } \\
\text { científica de la } \\
\text { investigación con } \\
\text { el tema }\end{array}$ & $\begin{array}{l}\text { Revistas } \\
\text { Autores }\end{array}$ \\
\hline $\begin{array}{l}\text { Emparejamiento } \\
\text { bibliográfico }\end{array}$ & $\begin{array}{l}\text { Analizar la } \\
\text { estructura del } \\
\text { conocimiento } \\
\text { científico } \\
\text { contenido en la } \\
\text { base }\end{array}$ & Países de origen \\
\hline
\end{tabular}

Cabe aclarar que la extracción de los términos se realizó mediante el software VOSviewer, entendiendo que la distancia entre dos términos se calcula mediante la fuerza de su asociación (Van Eck 
y Waltman, 2007), y al término como una secuencia de nombres en documentos de texto (Van Eck y Waltman, 2011). En general, cuanto más fuerte es la relación entre dos términos, menor es la distancia entre ellos en el mapa (Heersmink y otros, 2012).

\section{Visualización de resultados}

Las matrices generadas se cargaron en VOSviewer, donde en la creación de las representaciones gráficas se midió la similitud de los valores de co-ocurrencia y co-citación de las unidades analizadas, para lo que se aplicó el índice de similaridad Fuerza de Asociación $(F A)$, tal como lo describen Van Eck y Waltman (2010), basado en la normalización de la intensidad de las asociaciones de las parejas seleccionadas en la unidad de análisis. Con la aplicación del índice se obtuvo el peso (weight) de cada co-citación y co-ocurrencia, dando lugar a matrices normalizadas. Posteriormente, se posicionaron los nodos en un espacio bidimensional, de manera que los cercanos se consideraron como estrechamente relacionados. La técnica de mapeo VOSviewer permitió ejecutar diferentes algoritmos de clustering para con ellos posicionar y clasificar las co-ocurrencias y co-citaciones en grupos similares. A su vez, con el algoritmo de clustering de VOSviewer se incluyeron diferentes parámetros de resolución, según el valor considerado para su configuración, de tal forma que se obtuvieron diferentes niveles de agregación. En ese sentido, se consideró al clúster como un conjunto de nodos estrechamente relacionados, donde cada uno de ellos se integra a una red.
Por último, los diferentes grupos generados se representaron en mapas de redes etiquetados, en los que las diferentes unidades se mostraron en círculos o nodos y etiquetas conectadas. El tamaño de los nodos en el análisis de co-citación indica el número normalizado de citas recibidas por cada ítem, y el grosor de las líneas la fuerza de los vínculos. El vínculo y la proximidad entre dos ítems identifican la relación de citación o co-ocurrencia en su caso entre dos unidades de análisis. El color aleatorio de los nodos indica el grupo con el que cada ítem está asociado (términos en grupos temáticos).

\section{RESULTADOS Y DISCUSIÓN}

A continuación, se presentan los resultados considerando los indicadores de carácter descriptivo y aquéllos en los que se realizó análisis mediante técnicas bibliométricas.

\section{Análisis descriptivo}

\section{Producción}

A partir de los descriptivos obtenidos de los 1.764 documentos seleccionados se elaboró la Tabla III, donde se señala que los artículos publicados representan el trabajo de 5.143 autores firmantes pertenecientes a 2.955 instituciones, entre los cuales se observa una mayor presencia masculina tanto como primer autor, como del número total de autores $(67,1 \%)$. Por otro lado, la mayor parte de

Tabla III. Descriptivos de artículos revisados

\begin{tabular}{|c|c|c|c|}
\hline Criterio & Total & Hombres & Mujeres \\
\hline \multicolumn{4}{|l|}{ Artículos } \\
\hline $\mathrm{N}$ (1 $1^{\circ}$ autor) & 633 & $480(75,8 \%)$ & $153(24,2 \%)$ \\
\hline Autores (todos) & 5.143 & $3.454(76,1 \%)$ & $1.689(32,9 \%)$ \\
\hline \multicolumn{4}{|l|}{ Idioma } \\
\hline Inglés & 1. $458(82,6 \%)$ & & \\
\hline Español & $65(3,6 \%)$ & & \\
\hline Ruso & $62(3,5 \%)$ & & \\
\hline Francés & $40(2,2 \%)$ & & \\
\hline Alemán & $29(1,6 \%)$ & & \\
\hline \multicolumn{4}{|l|}{ Área de estudio* } \\
\hline Ciencias sociales & 704 & & \\
\hline Economía, Econometría y Finanzas & 441 & & \\
\hline Ciencia medioambiental & 374 & & \\
\hline Ciencias agrícolas y biológicas & 290 & & \\
\hline Ciencias de la Tierra y Planetarias & 253 & & \\
\hline
\end{tabular}

*Nota: Un mismo artículo puede corresponder a más de una área de estudio. 
Figura 1: Colaboración en base al número de autores por publicación.

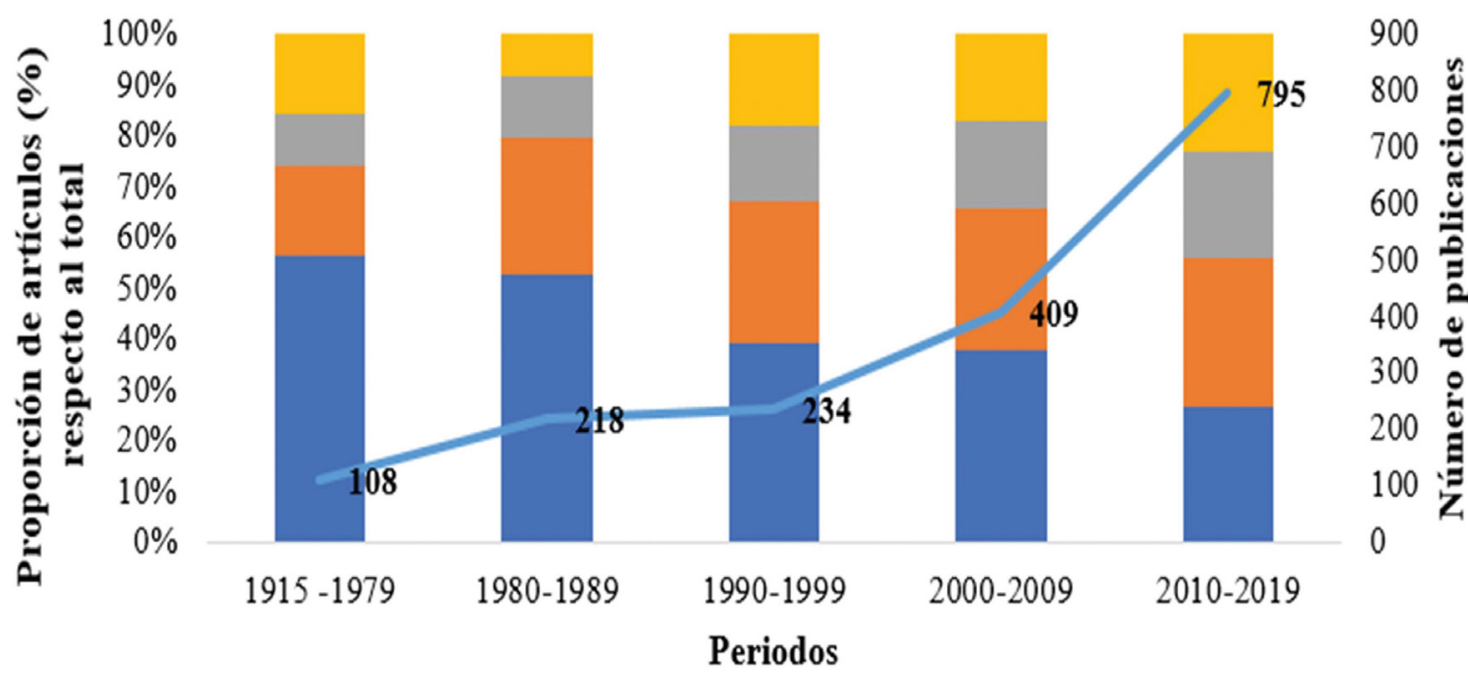

Uno Dos Tres Cuatro o más Total (escala derecha)

ellos ha sido publicado en inglés $(82,6 \%)$, con un número significativamente menor de trabajos publicados en español (9,9\%), y con predominancia de ciencias sociales como área de estudio.

\section{Colaboración}

En términos del grado de colaboración observado, en la Figura 1 se muestra el número de artículos distribuidos según el número de autores firmantes, a lo largo del periodo de estudio dividido en décadas a excepción del lapso inicial, donde por el escaso número de publicaciones se considera un lapso de tiempo más extenso. En general, sin considerar períodos, se observa mayor proporción de artículos firmados por un solo autor, y un menor número de documentos elaborados en colaboración.

Por otra parte, al comparar las proporciones en el número de autores durante los periodos se observa una transición en la distribución de porcentajes, ya que inicialmente la mayor parte de los documentos reflejaba un escaso nivel de colaboración entre autores, hacia un incremento posterior, materializado en un equilibrio en la proporción de artículos por número de autores firmantes observados en el último período. Esta transición genera un aumento progresivo de la proporción de artículos con mayor colaboración (firmados por dos o tres autores), junto a un incremento en el porcentaje de artículos con mayor colaboración (lo anterior, en números absolutos y relativos).

Con relación a naciones con mayor número de publicaciones en la temática, destacan Estados Unidos, Reino Unido y España (Figura 2). Para el caso de México, únicamente se encontraron 24 contribuciones. Finalmente, los artículos de esta base provienen mayoritariamente de personas afiliadas a instituciones de Países Bajos (Wageningen University \& Research), España (Universitat de Barcelona) y Rusia (Russian Academy of Sciences).

Figura 2: Países que publicaron sobre Especialización Productiva en el periodo 1915-2019

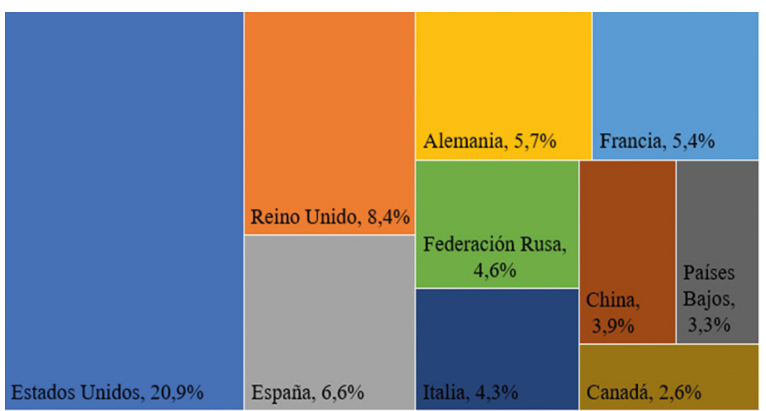

\section{Tendencias en el estudio de Especialización Productiva}

Con respecto al número de artículos publicados de 1915 - 2019, la muestra analizada presenta una tendencia con incremento progresivo durante el periodo 2010-2019 (Figura 3), situación que se relaciona con un incremento de estudios concernientes a temas vinculados con análisis territoriales. Cabe señalar que antes de 1920, únicamente se había publicado un artículo con la temática en cuestión 
Figura 3: Evolución de publicaciones referentes a Especialización Productiva para el periodo de 1915-2019.

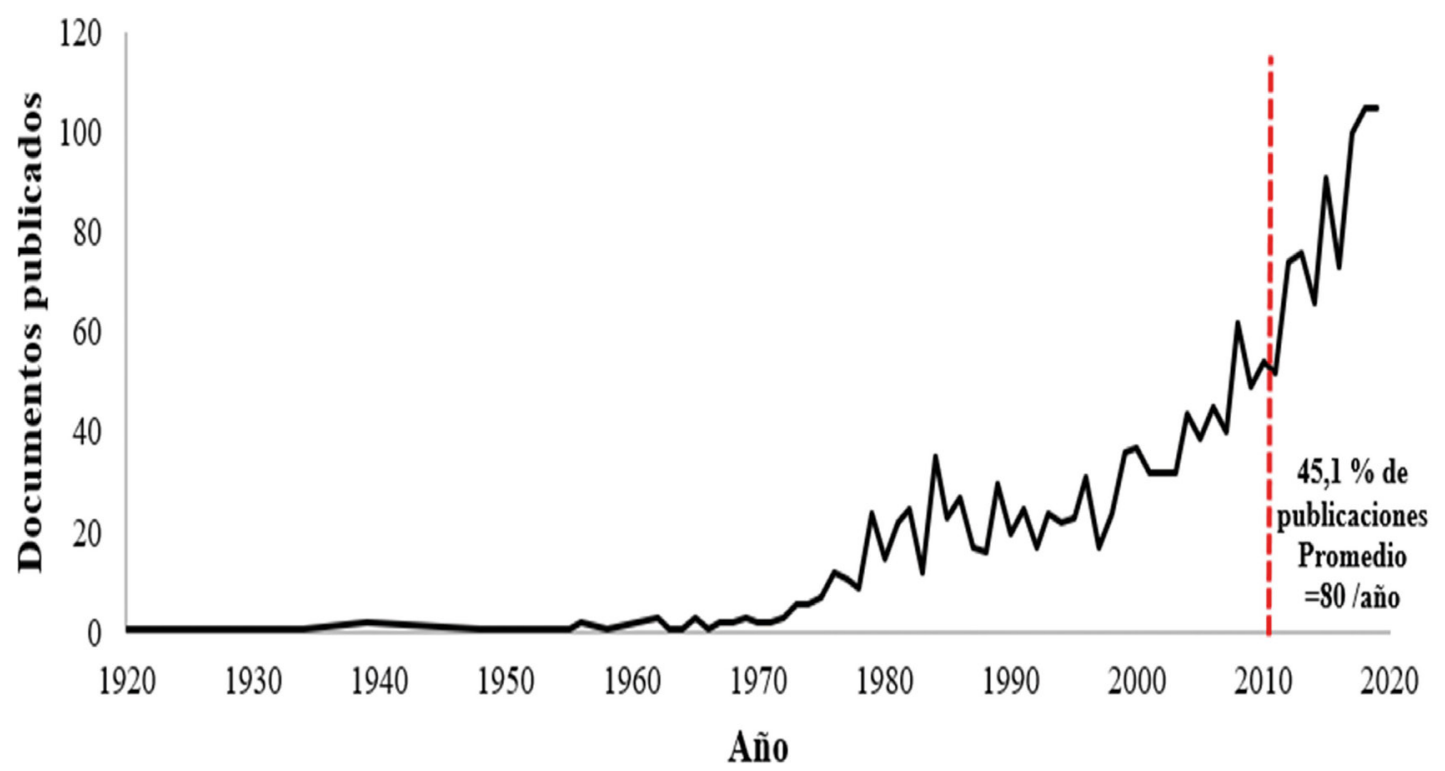

(1915 =1). Sin embargo, a partir de este año, comienza el abordaje de la misma, de manera constante y con tendencia creciente en la última década.

Una vez caracterizados los documentos, a continuación, se presentan los resultados obtenidos de la aplicación de técnicas bibliométricas.

\section{Análisis clúster}

\section{Co-ocurrencia}

A partir del listado de 34 términos considerados en esta técnica bibliométrica se realizó la caracte- rización de cada grupo, como base para la construcción del mapeo donde se representan las relaciones entre los mismos y su asociación en grupos temáticos (Figura 4). En ese sentido, se identificó que los términos que pertenecen al mismo grupo tienden a estar más estrechamente relacionados.

Cabe señalar, que la designación del nombre de cada uno de los conglomerados se realizó en función de la mayoría de las palabras clave que lo formaron. En dicho sentido, para este período de análisis se identificaron tres líneas de investigación presentes en la literatura revisada: i) especializa-

Figura 4: Mapa bibliométrico etiquetado de la red de co-ocurrencias con 34 términos seleccionados a partir de documentos referentes a Especialización Productiva para el periodo de 1915-2019.

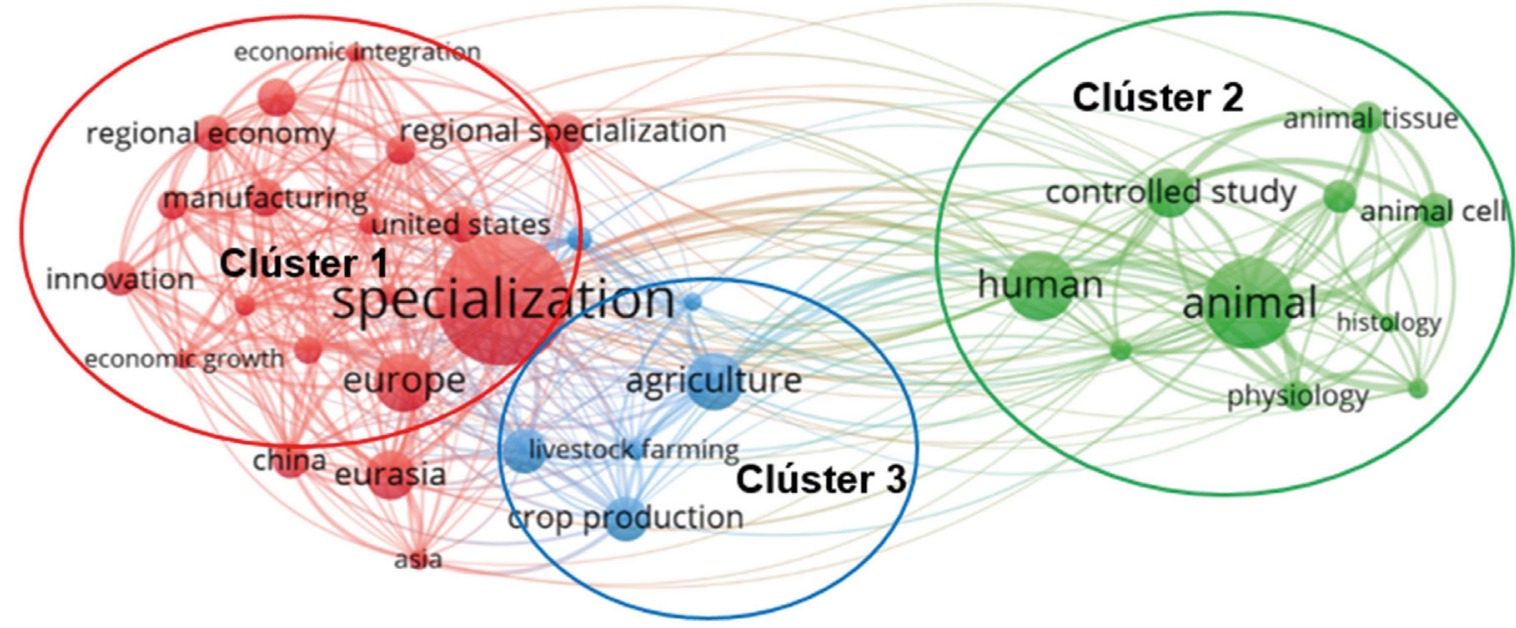


ción regional y crecimiento económico (color rojo); ii) neurociencia en humanos y animales (color verde); y iii) producción y prácticas agrícolas (color azul). Los grupos se conformaron de acuerdo con las palabras presentes en la Tabla IV.
En la Tabla $V$ se detallan algunas características de los documentos que mayores aportes brindaron para la caracterización del grupo temático.

Para mayor detalle, a continuación, se presentan los contenidos de los agregados conformados.

Tabla IV. Detalle de los grupos temáticos de co-ocurrencias

\begin{tabular}{|l|l|l|l|}
\hline $\begin{array}{c}\text { Clúster } \\
\text { (No y color) }\end{array}$ & \multicolumn{1}{|c|}{ Nombre } & \multicolumn{1}{c|}{$\begin{array}{c}\text { Artículos } \\
\text { englobados }\end{array}$} \\
\hline 1.- Rojo & $\begin{array}{l}\text { Especialización } \\
\text { regional y crecimiento } \\
\text { económico }\end{array}$ & $\begin{array}{l}\text { Specialization, agglomeration, Asia, China, economic } \\
\text { development, economic growth, economic integration, } \\
\text { employment, Eurasia, Europe, industrial production, } \\
\text { innovation, international trade, manufacturing, regional } \\
\text { development, regional economy, regional specialization, } \\
\text { United States. }\end{array}$ \\
\hline 2.- Verde & $\begin{array}{l}\text { Neurociencia en } \\
\text { humanos y animales }\end{array}$ & $\begin{array}{l}\text { Animal, animal cell, animal experiment, animal tissue, } \\
\text { comparative study, controlled study, cytology, histology, } \\
\text { human, physiology. }\end{array}$ \\
\hline 3.- Azul & $\begin{array}{l}\text { Producción y prácticas (25,3\%) } \\
\text { agrícolas }\end{array}$ & $\begin{array}{l}\text { Agricultural production, agriculture, crop production, land } \\
\text { use, livestock farming, Spain }\end{array}$ \\
\hline
\end{tabular}

Tabla V. Detalle del contenido de los grupos temáticos de co-ocurrencias

\begin{tabular}{|c|c|c|c|c|}
\hline Clúster & Autor, año & Documento & Revista & $\begin{array}{l}\text { País que } \\
\text { publica }\end{array}$ \\
\hline \multirow{3}{*}{1} & Kim, S (1995). & $\begin{array}{l}\text { Expansion of markets and the geographic } \\
\text { distribution of economic activities: The trends } \\
\text { in U. S. Regional manufacturing structure, } \\
\text { 1860-1987. }\end{array}$ & $\begin{array}{l}\text { Quarterly Journal } \\
\text { of Economics. }\end{array}$ & Reino Unido \\
\hline & De Gouvello (1999). & $\begin{array}{l}\text { Energy supply and agricultural specialisation } \\
\text { in the settlement frontiers in Southern } \\
\text { Amazonia [Approvisionnement energetique et } \\
\text { specialisation agricole des regions pionnieres: } \\
\text { L'Amazonie meridionale] }\end{array}$ & $\begin{array}{l}\text { Espace } \\
\text { Geographique }\end{array}$ & Francia \\
\hline & $\begin{array}{l}\text { Cheng y otros } \\
(2015) \text {. }\end{array}$ & $\begin{array}{l}\text { High-speed rail networks, economic } \\
\text { integration and regional specialisation in } \\
\text { China and Europe }\end{array}$ & $\begin{array}{l}\text { Travel Behaviour } \\
\text { and Society. }\end{array}$ & Países Bajos \\
\hline \multirow{3}{*}{2} & Elston, G (2003). & $\begin{array}{l}\text { The pyramidal neuron in occipital, temporal } \\
\text { and prefrontal cortex of the owl monkey } \\
\text { (Aotus trivirgatus): Regional specialization in } \\
\text { cell structure. }\end{array}$ & $\begin{array}{l}\text { European Journal } \\
\text { of Neuroscience. }\end{array}$ & Reino Unido \\
\hline & $\begin{array}{l}\text { Elston, Oga, } \\
\text { Okamoto, y Fujita } \\
(2010) \text {. }\end{array}$ & $\begin{array}{l}\text { Spinogenesis and pruning from early visual } \\
\text { onset to adulthood: An intracellular injection } \\
\text { study of layer III pyramidal cells in the ventral } \\
\text { visual cortical pathway of the macaque monkey. }\end{array}$ & Cerebral Cortex & Reino Unido \\
\hline & $\begin{array}{l}\text { Bianchi y otros } \\
\text { (2013). }\end{array}$ & $\begin{array}{l}\text { Dendritic morphology of pyramidal neurons } \\
\text { in the chimpanzee neocortex: Regional } \\
\text { specializations and comparison to humans. }\end{array}$ & Cerebral Cortex & Reino Unido \\
\hline \multirow{3}{*}{3} & $\begin{array}{l}\text { Billen, Le Noë y } \\
\text { Garnier (2018). }\end{array}$ & $\begin{array}{l}\text { Long-term changes in greenhouse gas } \\
\text { emissions from French agriculture and livestock } \\
\text { (1852-2014): From traditional agriculture to } \\
\text { conventional intensive systems }\end{array}$ & $\begin{array}{l}\text { Science of the } \\
\text { Total Environment }\end{array}$ & Países Bajos \\
\hline & Bengoa (2013). & Rural Chile Transformed: Lights and Shadows & $\begin{array}{l}\text { Journal of Agrarian } \\
\text { Change }\end{array}$ & Reino Unido \\
\hline & $\begin{array}{l}\text { Le Féon y otros } \\
\text { (2010). }\end{array}$ & $\begin{array}{l}\text { Intensification of agriculture, landscape } \\
\text { composition and wild bee communities: A large } \\
\text { scale study in four European countries }\end{array}$ & $\begin{array}{l}\text { Agriculture, } \\
\text { Ecosystems and } \\
\text { Environment }\end{array}$ & España \\
\hline
\end{tabular}




\section{Clúster 1: Especialización regional y crecimien- to económico}

Se destaca que es el grupo con más contribuciones y se caracteriza porque más que proporcionar concepto alguno del término especialización productiva, se centra en los procesos y elementos que influyen en ella y el desarrollo de este sector, señalando también ventajas y desventajas del proceso. En este sentido, sobresalen estudios que presentan evidencias sobre las tendencias a largo plazo en la especialización y localización regional basadas en explicaciones que parten de economías de escala y el modelo de Heckscher-Ohlin (Kim, 1995).

Se menciona que la lejanía de los mercados es un factor decisivo, puesto que agrega una dimensión estratégica al precio de insumos requeridos en la fase productiva (Gouvello, 1999). Por lo anterior, la proximidad es de vital importancia, ya que la colaboración interactiva será menos costosa y más fluida cuanto menor sea la distancia entre los participantes de determinada actividad. Por lo tanto, las aglomeraciones de la actividad económica relacionada no son solo reminiscencias de configuraciones espaciales anteriormente rentables, sino que actualmente se están recreando como resultado de una creciente demanda de transferencia rápida de conocimiento entre empresas (Malmberg y Maskell, 1997).

Por otro lado también destaca el vínculo existente entre la especialización regional y el proteccionismo local (Bai y otros, 2004), ya que existe una menor concentración geográfica en industrias donde los márgenes de impuestos y las acciones de propiedad estatal son altas, lo que refleja una mayor protección del gobierno local de estas industrias. Por último, el papel del transporte en el proceso de desarrollo económico e integración es considerado un área de controversia, se examinan los cambios en la accesibilidad con base en cambios en la especialización tanto para las principales ciudades como para sus zonas de influencia (Cheng y otros et al., 2015).

En este grupo temático, también se ha estudiado la evolución de la localización de producciones regionales, y la contribución de los procesos de Especialización Productiva en el crecimiento local, por lo que se analiza la relación especialización sectorial-desarrollo regional (Diniz y Carvalho, 2015). De igual forma, estos procesos se vinculan con la resiliencia productiva (Cuadrado-Roura y Maroto, 2016), donde ubican el comportamiento resiliente mediante el examen de la Especialización Productiva y sus efectos económicos en la mejora de la productividad regional como factores explicativos. Por otra parte, Capello y Perucca (2017) evalúan el impacto de la reestructuración industrial en el crecimiento económico regional, muestran que aquellas áreas capaces de reconvertir su
Especialización Productiva tanto en nuevos sectores como en actividades que agreguen mayor valor, logran el mejor desempeño económico. Finalmente se destaca que de las publicaciones correspondientes a México (24), el $50 \%$ de ellas se ajusta en este grupo temático.

\section{Clúster 2: Neurociencia en humanos y animales}

Es importante mencionar que este clúster cuenta con una amplia gama de publicaciones enfocadas en el campo de la neurociencia y es el más alejado de las ciencias sociales. Cabe señalar que más que describir procesos de especialización productiva, destaca por el uso de la variante Especialización Regional en casos prácticos, propios de esta área de conocimiento. En ese sentido Elston y otros (2010), se enfocan en el desarrollo de especializaciones regionales en la estructura neuronal de monos macaco, señalando que la experiencia visual puede influir en la maduración neuronal de diferentes formas en diferentes áreas corticales. De igual forma, analizan variaciones fenotípicas en células piramidales de la corteza cerebral de primates, destacando que dichas variaciones regionales no son exclusivas de primates, sino que se extienden entre las especies de mamíferos, es decir, la especialización regional en el fenotipo de las células piramidales no se limita a los primates (Elston y otros, 2006). En resumen, son estudios centrados en el análisis de la especialización regional de estructuras celulares piramidales de la corteza límbica, sensorial-motora y visual, sobre todo de monos vervet (Elston y otros, 2005), babuinos y monos búho (Elston, 2003).

En otros estudios también se enfatiza en especializaciones regionales de la morfología dendrítica de neuronas piramidales en la neocorteza de chimpancés comparadas con humanos (Bianchi y otros, 2013) y aspectos relacionados con la sensibilidad diferencial del tronco encefálico (Kasymov y otros, 2013).

\section{Clúster 3: Producción y prácticas agrícolas}

Se caracteriza por ser el grupo con menor número de contribuciones, y dentro de él se analiza la agricultura, especialización y diversificación productiva, en ese sentido, a partir de estimaciones del producto agrícola regional, se cuestiona el modelo tradicional de la producción agrícola (especialización versus diversificación), así como los factores que originan el proceso de Especialización Agrícola (Fonseca, 1995). En cuanto a tendencias sobre la elección ante dos escenarios, como son apertura y especialización, y mayor autonomía en escalas agrícola y regional, Billen y otros (2018), muestran que el escenario que generaliza prácticas agrícolas orgánicas y reconexión de sistemas de cultivo y ganadería, permite satisfacer la futura demanda nacional de alimentos al tiempo 
que exporta cantidades importantes al mercado internacional y garantiza una mejor calidad de aguas subterráneas.

Desde la perspectiva de sus prácticas en términos de insumos agrícolas (fertilización nitrogenada y pesticidas, densidad del ganado y tipos de cultivos), se destaca que los procesos de Especialización Agrícola influyen en el desarrollo y composición paisajística y riqueza faunística, ya que la intensificación agrícola genera efectos negativos en la riqueza de especies, abundancia y diversidad de insectos (Le Feón y otros, 2010). Así mismo, en la mayoría de las subcuencas del Báltico los problemas de eutrofización que impiden el logro de una buena situación ambiental tienen a las prácticas agrícolas especializadas como factores responsables de una gran parte de la lixiviación de nutrientes (Granstedt y otros, 2008). Por último, en cuanto a efectos derivados de estas prácticas se encuentra el empleo agrícola, ya que con base en una provincia chilena caracterizada por sus excelentes vinos de exportación, se señala que los procesos de especialización traen consigo mercados laborales estacionales, que emplean predominantemente trabajadoras cuyas precarias condiciones laborales contrastan marcadamente con el éxito de sus exportaciones (Bengoa, 2013).

Las prácticas de la Especialización Agrícola, también se vinculan con la antropometría (Koepke y Baten, 2008), ya que la especialización ganadera se relaciona con la estatura humana, debido a que, tanto la tierra per cápita como la especialización en ganadería lechera son factores determinantes en el incremento de la estatura, esto porque la proximidad a la producción de proteínas da paso a la baja en los precios de la leche, posibilitando un aumento en su consumo con consecuencias directas en el estado nutricional. A su vez, Roguet y otros (2015) analizan los patrones de ubicación recientes de la producción de leche, carne de res, cerdo y aves, a partir del análisis de los procesos de concentración geográfica y Especialización Productiva, concluyen que estos aspectos se relacionan con el desarrollo de ventajas comparativas medioambientales, estímulo a la conformación de economías de escala y de aglomeración, así como con el mejoramiento de la organización industrial de las cadenas de suministro.

En este grupo temático, la literatura también se centra en cuestiones relacionadas con el sector agroindustrial como perspectiva para el desarrollo de la arquitectura agrícola moderna, desde un aspecto educativo, abordando la temática de tipos de construcción posibles de desarrollar de acuerdo con la Especialización Agrícola y prácticas agrícolas, tales como complejos agroindustriales vinculados con plantas de producción y procesamiento, granjas ur- banas, objetos de agroturismo y asentamientos agrarios (Abdrassilova, 2016). Finalmente, se señala que la participación de México en este grupo es prácticamente nula, ya que únicamente cuenta con ocho contribuciones ajustadas a la temática.

De este modo, las evidencias presentadas confirman que los estudios de Especialización Productiva han tenido un desarrollo creciente, por lo cual ofrecen posibilidades para el análisis y comprensión de una heterogeneidad de fenómenos de diversa índole (culturales, económicos, sociales, ambientales y políticos). Para el caso de la economía mexicana, se evidencia la necesidad de desarrollar estudios en el sector agrícola, que pueden corresponderse con la política nacional, basada en la firma de tratados comerciales tendientes a estimular la concentración de economías agropecuarias en ámbitos territoriales locales y regionales. De igual forma, a modificar patrones de cultivo tradicionales, y a transformar la producción agropecuaria nacional al conformar nuevos ámbitos de Especialización Productiva.

\section{Co-citación de fuentes}

En la Figura 5 se presentan las fuentes con mayor nivel de citación (al menos 30 veces) del cam-

Figura 5: Mapa etiquetado de la red de co-citación de fuentes de documentos referentes a Especialización Productiva para el periodo de 1915-2019.

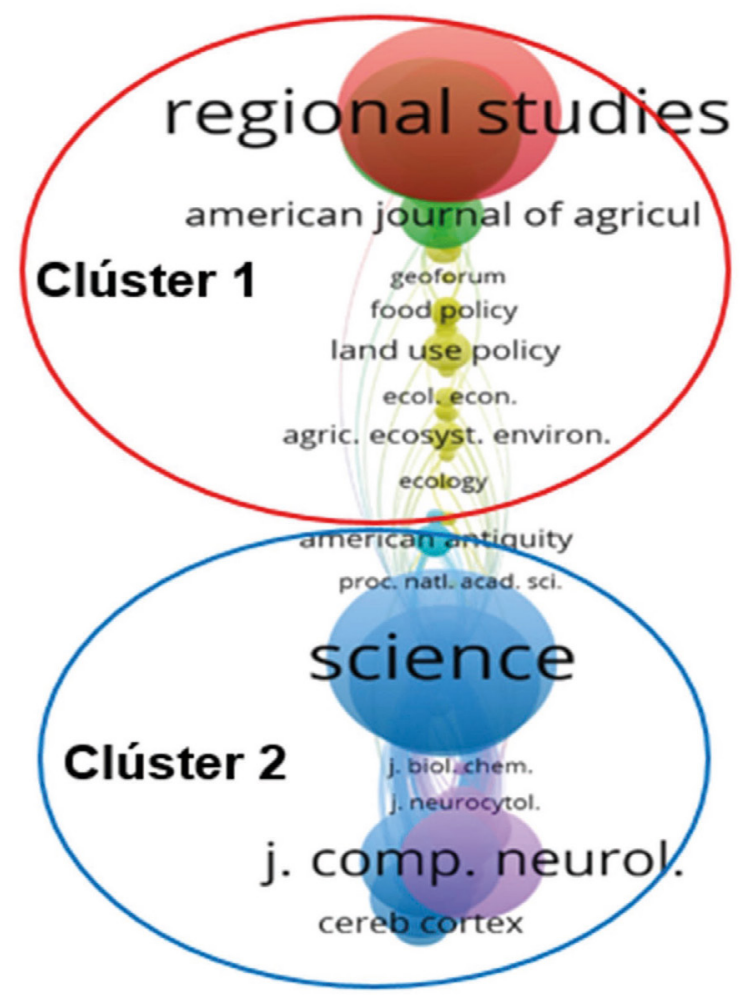


Tabla VI. Detalle de los grupos de co-citaciones de fuentes

\begin{tabular}{|c|c|c|c|c|}
\hline $\begin{array}{l}\text { Clúster } \\
\left(N^{\circ} \text { y color }\right)\end{array}$ & Disciplinas & Selección de co-citaciones & $\begin{array}{l}\text { Cite Score } \\
(2019)\end{array}$ & $\begin{array}{l}\text { JIF } \\
(2019)\end{array}$ \\
\hline \multirow{5}{*}{ 1-Rojo } & \multirow{5}{*}{$\begin{array}{l}\text { Economía, estudios } \\
\text { regionales y } \\
\text { geografía económica }\end{array}$} & American Journal of agricultural economics & 4,17 & 2,530 \\
\hline & & Land use policy & 6,20 & 3,682 \\
\hline & & Regional Studies & 3,94 & 3,074 \\
\hline & & Food policy & 7,00 & 3,788 \\
\hline & & Agriculture, ecosystems and environment & 8,10 & 3,230 \\
\hline \multirow{5}{*}{ 2-Azul } & \multirow{5}{*}{$\begin{array}{l}\text { Neurociencia } \\
\text { Bioquímica, Genética } \\
\text { y Biología Molecular }\end{array}$} & Science & 45,3 & 41,845 \\
\hline & & Journal of Comparative Neurology & 6,200 & 3,330 \\
\hline & & Cerebral cortex & 10,40 & 6,308 \\
\hline & & American antiquity & 3,70 & 1,980 \\
\hline & & $\begin{array}{l}\text { Proceedings of the National Academy of Sciences of } \\
\text { the USA }\end{array}$ & 15,70 & 6,900 \\
\hline
\end{tabular}

Nota. Cite Score: Scopus; JIF: Journal Impact Factor.

po de estudio y se observa que la red resultante mostró dos grandes grupos de publicaciones con patrones de citas similares.

Los nodos de mayor tamaño se correspondieron con las fuentes que presentaron un elevado número de citas, las que se identificaron a su vez con publicaciones que tienen un mayor nivel de asociación y similitud temática. Cada uno de los conglomerados se clasificó en diversas subdisciplinas (Tabla VI).

\section{Co-citación de autores}

En el análisis del listado de autores representativos del campo se observa que, la red de co-citación de autores mostró tres comunidades de investiga- dores incluidos dentro de la misma línea de investigación que abordan la temática Especialización Productiva (Figura 6).

De los autores señalados, también se presenta el Índice $h$ propuesto por Hirsch (2005) (Tabla VII).

\section{Emparejamiento bibliográfico por países}

Considerando aquellos países que han sido citados al menos quince veces, se obtuvo un total de cuarenta y cinco, donde sobresalen Estados Unidos (403 documentos y 12.740 citaciones), Reino Unido (161 documentos y 4.775 citaciones) y España (124 documentos y 1.852 citaciones), que como ya se ha comentado, son las naciones con mayor

Figura 6: Mapa etiquetado de la red de co-citación de autores de documentos referentes a Especialización Productiva para el periodo de 1915-2019.

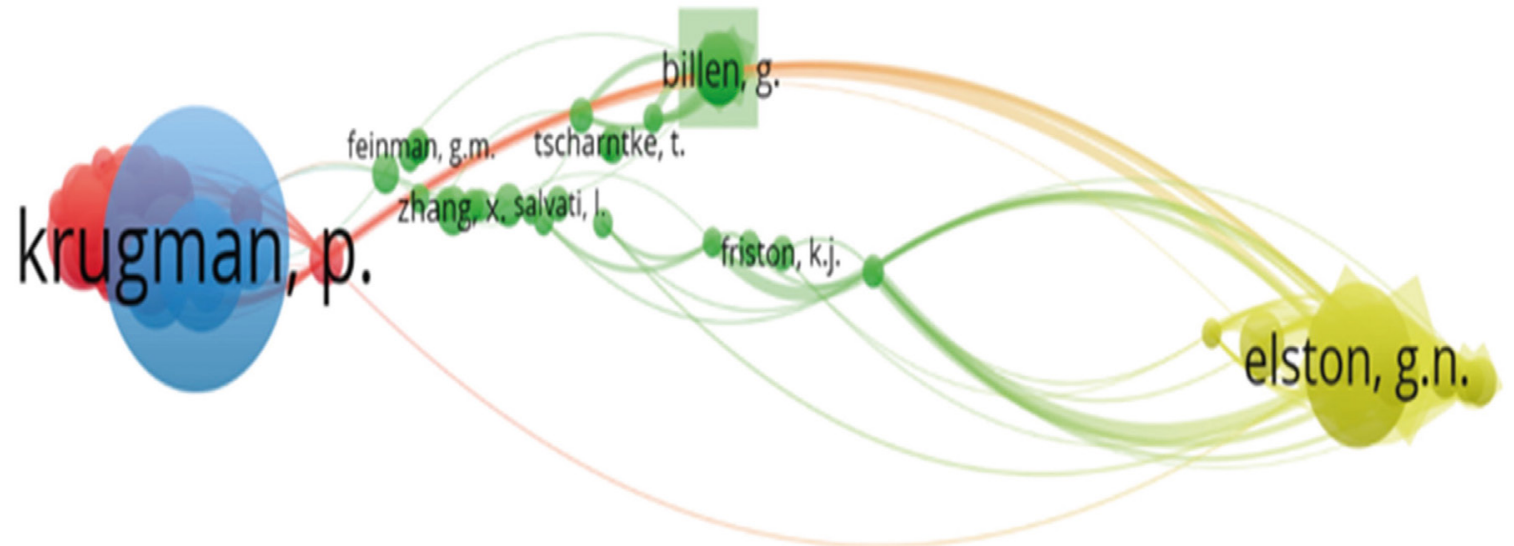


Tabla VII. Detalle de los grupos de co-citaciones de autores

\begin{tabular}{|c|c|c|c|c|}
\hline $\begin{array}{l}\text { Clúster } \\
\text { ( }{ }^{\circ} \text { y color) }\end{array}$ & Grupo temático & $\begin{array}{l}\text { Selección de } \\
\text { co-citaciones }\end{array}$ & Índice H & Adscripción \\
\hline \multirow{6}{*}{ 1.- Verde } & \multirow{3}{*}{$\begin{array}{l}\text { Econometría } \\
\text { espacial y teorías } \\
\text { del desarrollo y } \\
\text { crecimiento regional }\end{array}$} & Krugman, $\mathrm{P}$ & 45 & EE. UU. \\
\hline & & Becattini, G & 7 & $\begin{array}{l}\text { Universita degli Studi di Firenze, Florencia, } \\
\text { Italia }\end{array}$ \\
\hline & & Overman, H.G & 23 & $\begin{array}{l}\text { London School of Economics and Political } \\
\text { Science, Londres, Reino Unido }\end{array}$ \\
\hline & \multirow{3}{*}{$\begin{array}{l}\text { Ciencias agrícolas, } \\
\text { biológicas y medio } \\
\text { ambientales }\end{array}$} & Billen, G. & 62 & $\begin{array}{l}\text { Milieux Environnementaux, Transferts et } \\
\text { Interactions dans les Hydrosystèmes et les } \\
\text { Sols, Paris, Francia }\end{array}$ \\
\hline & & Garnier, J & 51 & $\begin{array}{l}\text { CNRS Centre National de la Recherche } \\
\text { Scientifique, Paris, Francia }\end{array}$ \\
\hline & & Lassaletta, L & 1 & Hospital Universitario La Paz, Madrid, España \\
\hline \multirow{3}{*}{ 2.- Café } & \multirow{3}{*}{$\begin{array}{l}\text { Neurociencia, } \\
\text { Bioquímica, Genética } \\
\text { y Biología Molecular }\end{array}$} & Elston, Guy N. & 32 & $\begin{array}{l}\text { Universidad de Queensland, Brisbane, } \\
\text { Australia }\end{array}$ \\
\hline & & Morrison John, $\mathrm{H}$ & 102 & $\begin{array}{l}\text { Universidad de California, Davis, Davis, } \\
\text { Estados Unidos. }\end{array}$ \\
\hline & & $\begin{array}{l}\text { Benavides- } \\
\text { Piccione, R. }\end{array}$ & 25 & CSIC - Instituto Cajal (IC) , Madrid, España \\
\hline
\end{tabular}

Figura 7: Mapa etiquetado de la red de emparejamiento bibliográfico por países, de documentos referentes a Especialización Productiva para el periodo de 1915-2019.

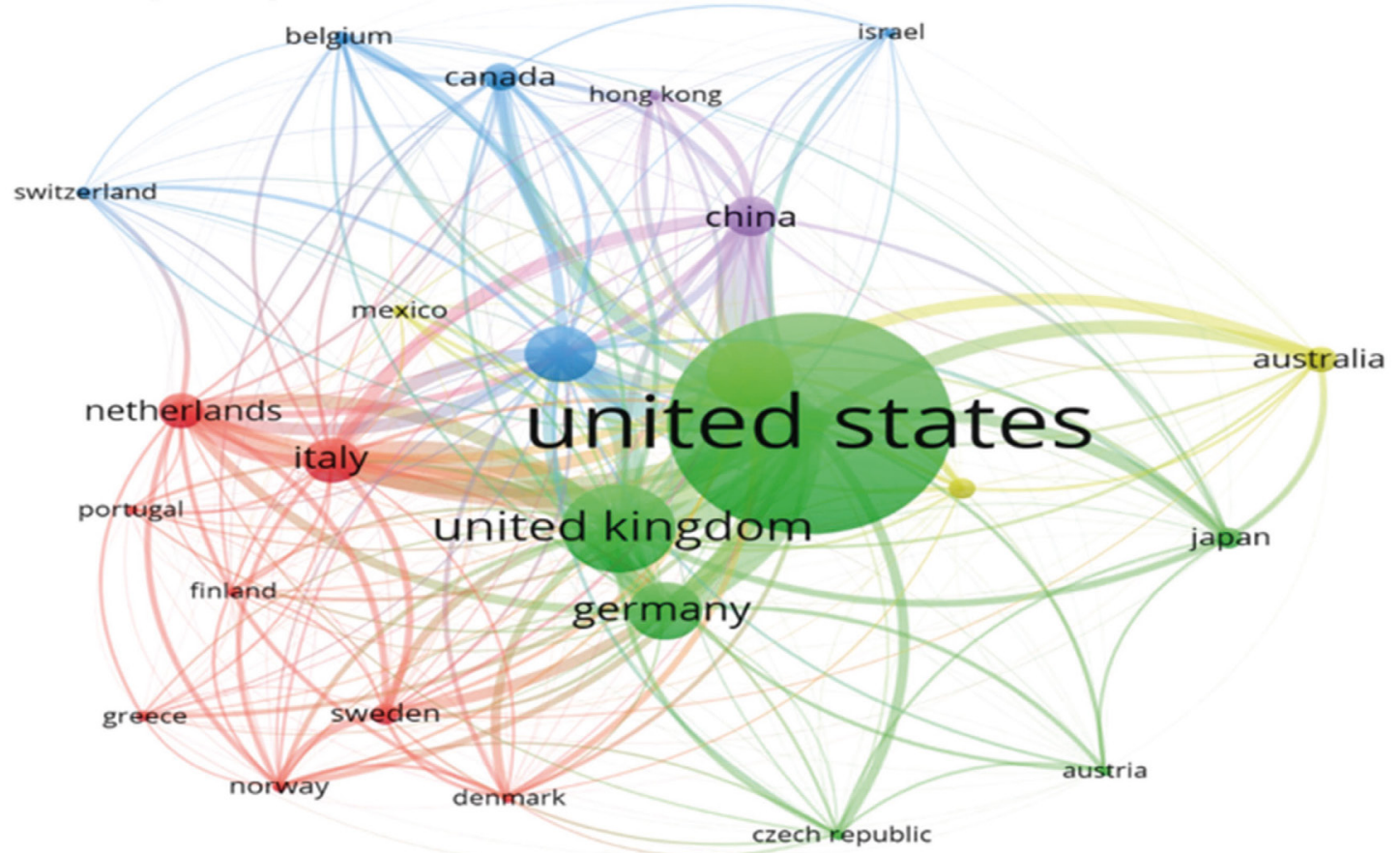

número de publicaciones en la temática (Figura 7). Cabe señalar que, en el caso particular de España, sobresale también por las instituciones a las cuales se encuentran adscritos los autores influyentes del tema de interés, tales como Maroto A, Maudos J, Pastor J, y Lassaletta L, así como por revistas de impacto entre las que se destacan la Revista Ciudad y Territorio, Estudios Territoriales, Investigaciones Regionales, Revista de Estudios Regionales, Cuadernos Geográficos, entre otras. Mientras que México cuenta con veintitrés documentos y ciento noventa y nueve citaciones. 
Tabla VIII. Detalle de los grupos de emparejamiento bibliográfico por países.

\begin{tabular}{|c|c|c|c|}
\hline $\begin{array}{l}\text { Clúster } \\
\left(N^{\circ} y\right. \\
\text { color) }\end{array}$ & Países & $\begin{array}{c}\text { Total de } \\
\text { documentos }\end{array}$ & $\begin{array}{l}\text { Total de } \\
\text { citaciones }\end{array}$ \\
\hline \multirow{4}{*}{ 1.- Rojo } & Italia & 83 & 2.021 \\
\hline & Dinamarca & 20 & 528 \\
\hline & $\begin{array}{l}\text { Finlandia } \\
\text { Grecia }\end{array}$ & 11 & 136 \\
\hline & $\begin{array}{l}\text { Países } \\
\text { Bajos }\end{array}$ & 64 & 1.569 \\
\hline \multirow{5}{*}{ 2.- Verde } & Alemania & 107 & 2.495 \\
\hline & Reino Unido & 161 & 4.775 \\
\hline & $\begin{array}{l}\text { Estados } \\
\text { Unidos }\end{array}$ & 403 & 12.740 \\
\hline & $\begin{array}{l}\text { Republica } \\
\text { Checa }\end{array}$ & 18 & 221 \\
\hline & Austria & & \\
\hline \multirow{5}{*}{ 3.- Azul } & Francia & 103 & 2.267 \\
\hline & Canadá & 50 & 902 \\
\hline & Suiza & & \\
\hline & Bélgica & 26 & 592 \\
\hline & Israel & & \\
\hline \multirow{4}{*}{ 4.- Amarillo } & España & 124 & 1.852 \\
\hline & Australia & 48 & 1.072 \\
\hline & Brasil & & \\
\hline & México & 23 & 199 \\
\hline \multirow{2}{*}{ 5.- Morado } & China & 75 & 1.079 \\
\hline & Hong Kong & 12 & 434 \\
\hline
\end{tabular}

Como se observa, la red de emparejamiento bibliográfico mostró cinco grupos de países cuyas producciones tienen referencias bibliográficas comunes. Éstos se resumen en la Tabla VIII.

\section{CONCLUSIONES}

Las líneas de investigación abordadas en torno a la temática de Especialización Productiva en general, a lo largo del periodo de análisis, se enfocan a en describir factores detonantes de estos procesos, así como sus repercusiones en el crecimiento de intercambios comerciales en un escenario de globalización. En una menor proporción enfatizan en su medición a través de múltiples enfoques metodológicos con fines de generación de insumos para la formulación de políticas públicas. Sin embargo y pese a que en el sector primario los efectos de estos procesos se maximizan por la configuración de un orden internacional centralizado y sustenta- do en la división internacional del trabajo, donde los países desarrollados incursionan en cultivos de vanguardia, los estudios con fines de medición de niveles de Especialización Agrícola en casos específicos son prácticamente nulos.

El análisis permitió identificar líneas de investigación y referentes empíricos dentro de la temática de Especialización Productiva a considerar en futuras investigaciones, además se comprobó que los sectores que transitan por esta senda son múltiples, siendo mayormente analizado el industrial, debido a que la propiedad corporativa es más prevaleciente, seguido de la neurociencia que destaca por el uso de la variante Especialización Regional, aunque éste es aplicado en temas de gran contraste. Esto genera vacíos de conocimiento destinados al estudio del modelo en el sector agropecuario, como ocurre especialmente en el caso de México; por ello, las futuras investigaciones se deben centrar en niveles de especialización en ámbitos regionales, así como cambios detectados en dichos perfiles. De igual forma, se podría profundizar en la importancia de cultivos intensivos que surgen como especializaciones en zonas menos desarrolladas, el análisis de factores explicativos según su intensidad y sobre todo la adaptación de enfoques metodológicos para su aplicación en el sector agrícola.

Finalmente, resulta necesario indagar cuáles son los requerimientos de información para analizar los procesos de Especialización Productiva y Especialización Agrícola. Para el contexto mexicano, el escenario legislativo y desarrollo de regiones con Especialización Productiva acompañado de políticas de gobierno que promueven este modelo, a través del desarrollo de cadenas agroalimentarias (estrategia prioritaria en la política agropecuaria), representan un motivo para el análisis de la Especialización Agrícola del país, con la finalidad de entender su dinámica y aprovechar ventanas de oportunidades derivadas de la misma.

\section{REFERENCIAS}

Abbasi, A.; Altmann, J. (2010). A social network system for analyzing publication activities of researchers. TEMEP Discussion Papers.

Abdrassilova, G. (2016). The agro-industrial sector as a perspective direction for the development of Kazakhstan architecture: An educational aspect. Global Journal of Engineering Education, 18(3).

Arias, J. A. ; Fortich, F. J. (2010). El panorama teórico de la economía regional y los modelos de análisis territorial. Finanzas y Política Económica, 2(2), 9-26.

Bai, C.; Du, Y.; Tao, Z.; Tong, S. (2004). Local protectionism and regional specialization: Evidence from China's industries. Journal of International Economics, $63(2)$. 
Becattini, G. (2006). Vicisitudes y potencialidades de un concepto: el distrito industrial. Revista Economía Industrial, 359, 21-27.

Bengoa, J. (2013). Rural Chile Transformed: Lights and Shadows. Journal of Agrarian Change.

Beretta, V.; Lobato, J.; Mielitz, C. (2002). Produtividade e Eficiência Biológica de Sistemas de Recria e Engorda de Gado de Corte no Rio Grande de Sul. Revista Brasileira de Zootecnia, 31(2).

Bianchi, S.; Stimpson, C.; Bauernfeind, A.; Schapiro, S.; Baze, W.; McArthur, M.; Bronson, E.; Hopkins, W.D.; Semendeferi, K.; Jacobs, B.; Hof, P.; Sherwood, C. (2013). Dendritic morphology of pyramidal neurons in the chimpanzee neocortex: Regional specializations and comparison to humans. Cerebral Cortex, 23(10).

Billen, G.; Le Noë, J.; Garnier, J. (2018). Two contrasted future scenarios for the French agro-food system. Science of The Total Environment, 63 (7).

Boisier, S. (1980). Técnicas de Análisis Regional con Información Limitada. CEPAL-ILPES.

Capello, R.; Perucca, G. (2017). Industrial restructuring in CEE regions: determinants of regional growth in the accession and in the crisis period. Journal of BalticStudies, 48(3).

Cheng, Y.; Loo, B. P.;Vickerman, R. (2015). High-speed rail networks, economic integration and regional specialisation in China and Europe. Travel Behaviour and Society, 2(1).

Cuadrado-Roura, J.; Maroto-Sánchez, A. (2012). Analysis of the process of regional specialization in services in Spain. Revista Latinoamericana de Estudios Urbano Regionales, 38(114).

Cuadrado-Roura, J.; Maroto, A. (2016). Unbalanced regional resilience to the economic crisis in Spain: A tale of specialisation and productivity. Cambridge Journal of Regions Economy and Society, 9(1).

Diniz, F.; Carvalho, M. (2015). Productive activities of the Portuguese nuts III regions: Evolution analysis of location and regional specialization. Revista Brasileira de Gestao e Desenvolvimento Regional, 11(2).

ELSEVIER. (2016). Métricas CiteScore: un nuevo estándar de impacto de citas en revistas. https://www.elsevier.com/solutions/scopus/how-scopus-works/metrics

Elston, G. (2003). The pyramidal neuron in occipital, temporal and prefrontal cortex of the owl monkey (Aotus trivirgatus): Regional specialization in cell structure. European Journal of Neuroscience, 17(6).

Elston, G.; Benavides-Piccione, R.; Elston, A.; Manger, P.; DeFelipe, J. (2005). Regional specialization in pyramidal cell structure in the limbic cortex of the vervet monkey (Cercopithecus pygerythrus): An intracellular injection study of the anterior and posterior cingulate gyrus. Experimental Brain Research, 167(3).

Elston, G.; Elston, A.; Freire, M. A.; Gomes-Leal, W.; Dias, I. ; Pereira, J. A. ; Silveira, L. C.; Picanço-Diniz, C. (2006). Specialization of pyramidal cell structure in the visual areas V1, V2 and V3 of the South American rodent, Dasyprocta primnolopha. Brain Research, 1106(1).

Elston, G.; Oga, T.; Okamoto, T.; Fujit, I. (2010). Spinogenesis and pruning from early visual onset to adulthood: An intracellular injection study of layer III pyramidal cells in the ventral visual cortical pathway of the macaque monkey. Cerebral Cortex, 20(6).

Fonseca, A. (1995). Agriculture, specialisation and diversification of produce: the Portuguese experience in the "Region of Latifundia", 1850-1910. An interpretation. Revista de Agricultura e Historia Rural, 9.

Garfield, E. (1998). Mapping the world of science. The Scientist.

Garofoli, G. (1986). Áreas de especialización productiva y pequeñas empresas en Europa. Documentos de Análisis Geográfica, 8(9), 143-172.

Gómez, I.; Bordons Gangas, M. (1996). Limitaciones en el uso de los indicadores bibliométricos para la evaluación científica. Política Científica, 46.

Granstedt, A.; Schneider, T.; Seuri, P.;Thomsson, O. (2008). Ecological Recycling Agriculture to Reduce Nutrient Pollution to the Baltic Sea. Biological Agriculture \& Horticulture, 26(3).

Heersmink, R.; Van den Hoven, J.; Van Eck, N.; Van den Berg, J. (2012). Bibliometric Mapping of Computer and Information Ethics. Ethics and Information Technology, 13(3).

Hirsch, J. (2005). An index to quantify an individual's scientific research output. Proceedings of the National Academy of Sciences, 102(46).

Jimenez, B. (1993). Productividad en investigación del docente universitario. Revista Espacios Digitales, 14(3).

Kasymov, V. ; Larina, O. ; Castaldo, C. ; Marina, N.; Patrushev, M.; Kasparov, S.; Gourine, A. (2013). Differential sensitivity of brainstem versus cortical astrocytes to changes in $\mathrm{pH}$ reveals functional regional specialization of astroglia. Journal of Neuroscience, 33(2).

Kim, S. (1995). Expansion of markets and the geographic distribution of economic activities: The trends in U. S. Regional manufacturing structure, 1860-1987. Quarterly Journal of Economics, 110(4).

Koepke, N.; Baten, J. (2008). Agricultural specialization and height in ancient and medieval Europe. Explorations in Economic History, 45(2).

Le Feón, V.; Schermann-Legionnet, A.; Delettre, Y.; Aviron, S.; Billeter, R.; Bugter, R.; Hendrickx, F.; Burel, F. (2010). Intensification of agriculture, landscape composition and wild bee communities: A large scale study in four European countries. Agriculture, Ecosystems \& Environment, 137.

Malmberg, A.; Maskell, P. (1997). Towards an explanation of regional specialization and industry agglomeration. European Planning Studies, 5(1).

Marshall, A. (1920). Industry and Trade. (Vol. 83). Macmillan. https://doi.org/10.2307/2341084

Marshall, F. (1990). Origins of Specialized Pastoral Production in East Africa. American Anthropologist, 92(4).

Md Khudzari, J.; Kurian, J.; Tartakovsky, B.; Vijaya- Raghavan, G. (2018). Bibliometric analysis of global research trends on microbial fuel cells using Scopus database. Biochemical Engineering Journal, 136(15). 
Montero, I.; León, O. (2007). A guide for naming research studies in Psychology. International Journal of Clinical and Health Psychology, 7.

Ortega-Priego, J.:Aguillo, I. F. (2006). Análisis de co-enlaces: una aproximación teórica. El Profesional de la Información, 15(4).

Qin, Y.; Zhang, X. (2016). The Road to Specialization in Agricultural Production : Evidence from Rural China. World Development, 77, 1-16. https://doi.org/10.1016/j.worlddev.2015.08.007

Rebollar,R.A.: Rebollar, R.S.; Gómez, T.; Hernández, J.; Gónzalez, F. de J. (2016). Crecimiento y especialización regional del sector pecuario en México, 1994 a 2013. Revista Mexicana de Ciencias Pecuarias, 7(3), 391-403.

Roguet, C.; Gaigné, C.; Chatellier, V.; Cariou, S.; Carlier, M.; Chenut, R.; Daniel, K.; Perrot, C. (2015). Regional specialization and concentration of European livestock:
Situation and explanatory factors. Productions Animales, 28(1).

Sanz, C. E. (2000). Proyecto docente para la provisión de una plaza de Catedrático de Universidad sobre Bibliometría. Universidad Carlos III.

Thomson, R. (2019). El factor de impacto de Clarivate Analytics. http://thomsonreuters.com/products_services/science/free/essays/impact_factor/?elq $=8002 \mathrm{fc}-$ ce746d49ef968516f29d825087

Van Eck, J.; Waltman, L. (2007). VOS: A new method for visualizing similarities between objects. Advances in Data Analysis.

Van Eck, N.; Waltman, L. (2010). Software survey:Vosviewer, a computer program for bibliometric mapping. Scientometrics.

Van Eck, N.; Waltman, L. (2011). Text mining and visualization using VOSviewer. ISSI Newsletter, 7(3). 